ARTICLE

Received 11 Jun 2014 | Accepted 8 Sep 2014 | Published 22 Oct $2014 \quad$ DOI: 10.1038/ncomms6183

\title{
Reduced-dimensionality-induced helimagnetism in iron nanoislands
}

\author{
S.-H. Phark' , J.A. Fischer ${ }^{1,2}$, M. Corbetta ${ }^{1}$, D. Sander ${ }^{1}$, K. Nakamura ${ }^{3}$ \& J. Kirschner ${ }^{1}$
}

Low-dimensionality in magnetic materials often leads to noncollinear magnetic order, such as a helical spin order and skyrmions, which have received much attention because of envisioned applications in spin transport and in future data storage. Up to now, however, the real-space observation of the noncollinear magnetic order has been limited mostly to systems involving a strong spin-orbit interaction. Here we report a noncollinear magnetic order in individual nanostructures of a prototypical magnetic material, bilayer iron islands on $\mathrm{Cu}$ (111). Spin-polarized scanning tunnelling microscopy reveals a magnetic stripe phase with a period of $1.28 \mathrm{~nm}$, which is identified as a one-dimensional helical spin order. Ab initio calculations identify reduced-dimensionality-enhanced long-range antiferromagnetic interactions as the driving force of this spin order. Our findings point at the potential of nanostructured magnets as a new experimental arena of noncollinear magnetic order stabilized in a nanostructure, magnetically decoupled from the substrate.

\footnotetext{
${ }^{1}$ Max-Planck-Institut für Mikrostrukturphysik, Weinberg 2, Halle 06120, Germany. ${ }^{2}$ Laboratório de Filmes Finos e Superfícies, Departamento de Física, Universidade Federal de Santa Catarina, Florianópolis, Santa Catarina 88040-900, Brazil. ${ }^{3}$ Department of Physics Engineering, Mie University, Tsu, Mie 5148507, Japan. Correspondence and requests for materials should be addressed to S.-H.P. (email: shphark@gmail.com).
} 
T he magnetic order in solid depends on subtle details of the atomic structure and resulting distribution of the exchange interaction, which occasionally result in noncollinear magnetic order $(\mathrm{NCMO})^{1,2}$, where the spins are neither parallel nor antiparallel. The details of the spin order in NCMO may result from different contributions, such as competing exchange interaction ${ }^{3-5}$, Ruderman-Kittel-Kasuya-Yosida (RKKY) interaction, spin-orbit interaction $(\mathrm{SOI})^{6-9}$, quantum fluctuation of antiferromagnetic (AFM) order ${ }^{10}$ and Fermisurface nesting ${ }^{11-13}$, or their mutual interactions. So far, NCMO's in the bulk ${ }^{1,2}$ and at surfaces ${ }^{6-9}$ of materials exhibiting strong SOI have been intensively studied. SOI, in conjunction with broken inversion symmetry, induces a Dzyaloshinskii-Moriya interaction ${ }^{14,15}$ (DMI), which drives the formation of NCMO, provided its magnitude is comparable to the nearest-neighbour (NN) exchange interaction. Another
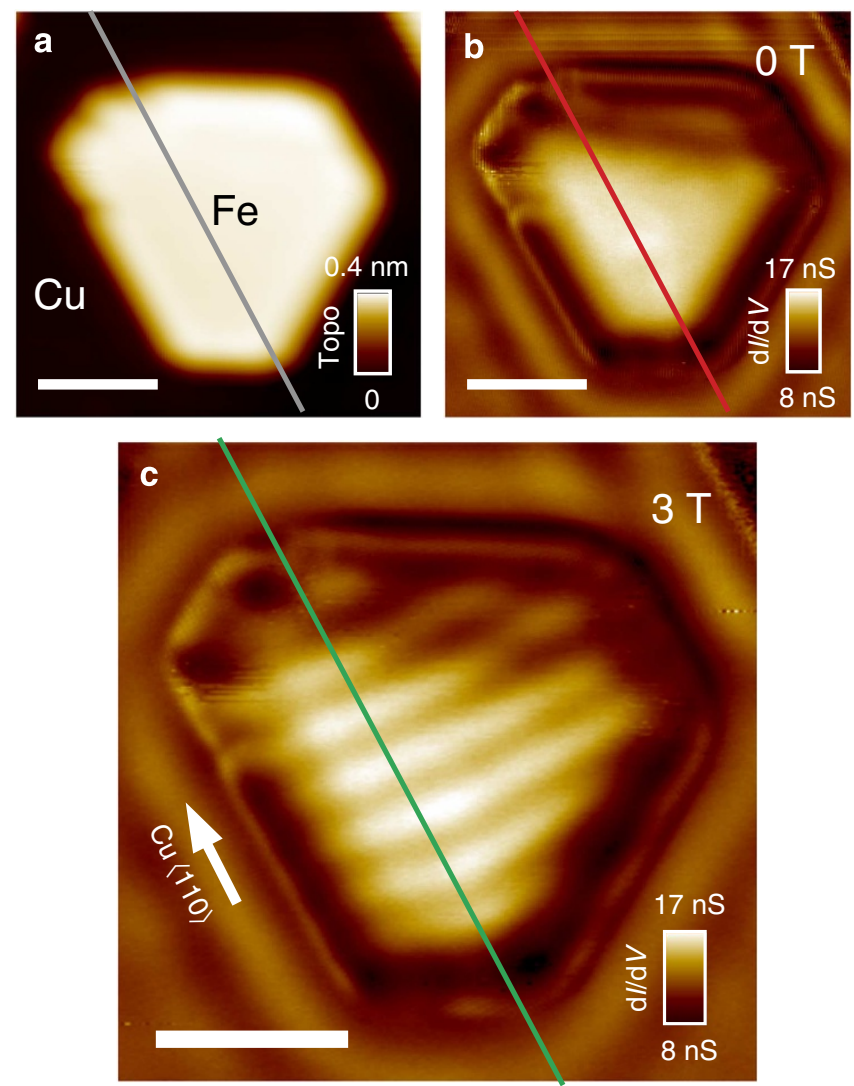

d

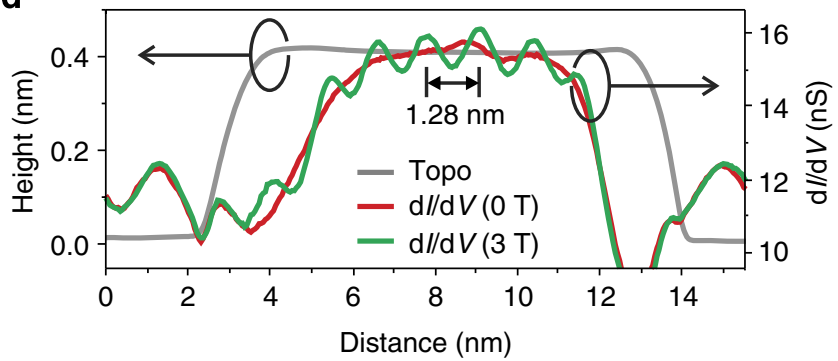

Figure 1 | Spin-polarized differential conductance map of an Fe island.

(a) Constant-current STM image of a bilayer Fe island on $\mathrm{Cu}(111)$

$\left(V_{b}=-0.3 \mathrm{~V}\right.$ and $\left.I_{\text {set }}=3 \mathrm{nA}\right)$. (b,c) Differential conductance $\mathrm{d} / / \mathrm{dV}$ images of the island in (a) at an external magnetic field of OT (b) and $3 T$

$\left(\mathbf{c} ; V_{\mathrm{b}}=-0.3 \mathrm{~V}\right.$ and $I_{\mathrm{Set}}=3 \mathrm{nA}$ ). (d) Profiles along grey, red and green lines in a-c, respectively. Scale bars in a-c indicate $4 \mathrm{~nm}$. common origin for NCMO involves long-range AFM interactions, which are of comparable size to the short-range ferromagnetic (FM) interaction ${ }^{16}$. This mechanism has been described within the framework of the Heisenberg exchange model $H=-\sum_{\mathrm{i}, \mathrm{j}} J_{\mathrm{ij}} \mathbf{S}_{\mathrm{i}} \cdot \mathbf{S}_{\mathrm{j}}{ }^{3-5}$, where $J_{\mathrm{ij}}$ is the exchange coupling between the pair of spins $\mathbf{S}_{i}$ and $\mathbf{S}_{j}$ at lattice sites $\mathrm{i}$ and $\mathrm{j}$, respectively.

NCMO in bulk phases of $3 d$ itinerant magnetic materials under a drastic change of the atomic volume by few ten $\%$ has been predicted by theory ${ }^{11}$. However, in special cases, as observed for $\mathrm{Fe}$, a structural transition from bcc $(\alpha-\mathrm{Fe})$ to fcc $(\gamma-\mathrm{Fe})$ mediates also a change of magnetic order from FM to AFM, respectively. Structure-related changes of magnetic order are promising for inducing $\mathrm{NCMO}^{17,18}$. The reduced dimensionalities and symmetries of magnetic nanostructures (MNs), supported on a surface are viable to induce novel magnetic states, which deviate from the respective bulk. This is because of the following: (1) lattice mismatch and epitaxial strain; (2) structural relaxation at interfaces; and (3) structural relaxation induced by finite lateral size and broken in-plane symmetry. These aspects lead to non-bulk-like atomic structures, which in turn modify the spindependent electronic properties of $\mathrm{MN}$, resulting in a possible driving force towards NCMO. So far, however, only theory has predicted NCMO in substrate-supported $\mathrm{MNs}^{4,5,19}$.

Here we report spin-polarized scanning tunnelling microscopy and spectroscopy (SP-STM/S) studies, combined with ab initio spin-density functional calculations, of a helical spin order (spin helix; $\mathrm{SH}$ ) in bilayer (BL) Fe nanoislands on $\mathrm{Cu}(111)$. It has been proposed that an extended $\mathrm{BL} F$ film on $\mathrm{Cu}(111)$ favours fcc stacking ('Fe-a' phase) with a prediction of AFM spin ordering ${ }^{20}$. In contrast, $\mathrm{BL} \mathrm{Fe}$ nanoislands on $\mathrm{Cu}(111)$ with a reduced lateral size $(<\sim 10 \mathrm{~nm})$ predominantly show an intriguing lattice structure, which favours a bridge-site-stacking of topmost $\mathrm{Fe}$ atoms ${ }^{20,21}$ ('Fe-b' phase). $\mathrm{Fe}$ is a prototypical example for studying the impact of structural relaxations on the exchange interaction, because of the pronounced interatomic distance dependence of the magnetic ordering ${ }^{17,18}$. This is also corroborated by the prediction of an $\mathrm{SH}$ in free-standing monolayers (MLs) of bcc-Fe(110) in theory ${ }^{12}$. In view of a change of spin order, driven by subtle structural changes, the $\mathrm{Fe} / \mathrm{Cu}(001)$ system is a prototypical example for extended $\mathrm{Fe}$ layers $^{22-24}$. We exploit that $\mathrm{Fe}$ in bridge-site-stacking is stabilized only in $\mathrm{nm}$-small BLr Fe islands on $\mathrm{Cu}(111)$, which plays a pivotal role of the formation of the $\mathrm{SH}$ reported here. In these respects, together with a negligibly small SOI of $\mathrm{Fe}$ and $\mathrm{Cu}$, the $\mathrm{Fe}-\mathrm{b}$ phase serves as a prototypical test case to study NCMO localized in a nanostructure, which is almost free from substrateinduced magnetic interactions.

\section{Results}

Spin-dependent differential conductance of $\mathrm{Fe}$ nanoislands. The SP-STM/S measurements were carried out at a temperature of $10 \mathrm{~K}$ and in an external magnetic field normal to the sample surface (see Methods section). Figure la shows a constant-current STM (CC-STM) topographic image of a typical Fe island, where the line profile reveals a height of $4 \AA$ (grey curve in Fig. 1d), indicative of a biatomic layer. The STS spectra identify the island as the structural phase $\mathrm{Fe}^{-20,21}$ (Supplementary Fig. 1; Supplementary Note 1). Figure $1 \mathrm{~b}, \mathrm{c}$ shows the differential conductance $\mathrm{d} I / \mathrm{d} V$ images measured at 0 and $3 \mathrm{~T}$ at $10 \mathrm{~K}$, respectively, of the island shown in Fig. 1a. The differential conductance image at $3 \mathrm{~T}$ shows a periodic stripe contrasts with a wavelength of $1.28 \mathrm{~nm}$ and a wave vector along the $\langle 110\rangle$ direction of the $\mathrm{Cu}$ substrate $(\approx \mathrm{Fe}\langle 110\rangle)$, which is absent in the differential conductance image at $0 \mathrm{~T}$. The tip shows a non-zero magnetization at $0 \mathrm{~T}$ (Supplementary Fig. 1; 
Supplementary Note 2), which indicates that the island does not present a measurable periodic magnetic order at $0 \mathrm{~T}^{25}$.

Figure 2a,b shows the differential conductance images of pure $\mathrm{Fe}$ islands and a Co island in circumferential contact with an $\mathrm{Fe}$ $\mathrm{BL}$ rim (Fe|Co island) ${ }^{26}$ (see also Methods section), respectively, where $\mathrm{Co}, \mathrm{Fe}-\mathrm{a}$ and $\mathrm{Fe}-\mathrm{b}$ are identified by STS measurements (Supplementary Fig. 1; Supplementary Note 1). The stripe patterns are observed also in the Fe-b phases of the $\mathrm{Fe}$ rim in $\mathrm{Fe} \mid \mathrm{Co}$ islands, with the same wavelength and orientation as in pure $\mathrm{Fe}-\mathrm{b}$ islands. Interestingly, the stripes show three distinct directions as indicated by the arrows (labels 1, 2 and 3 ). Correspondingly, the wave vectors of the stripe patterns are oriented along $\mathrm{Cu}\langle 110\rangle$. These three orientations of the stripe pattern are correlated with the three stacking possibilities of the topmost $\mathrm{Fe}$ atoms in the $\mathrm{Fe}-\mathrm{b}$ phase, as shown in the hard sphere model of Fig. 2c. For all three orientations, we measure a wavelength of the stripe patterns of $1.28 \pm 0.01 \mathrm{~nm}$, which is constant, irrespective of island size, shape and bias voltage. A striking aspect is the observation of the stripes at $0 \mathrm{~T}$ in the fielddependent differential conductance images of a $\mathrm{Fe} \mid \mathrm{Co}$ island (Fig. 2b) in contrast to a pure Fe island (Fig. 1). The Co core of the island in Fig. 2b shows a bistable magnetization state with a switching field of $0.75 \pm 0.05 \mathrm{~T}$. We propose that the remanent

a

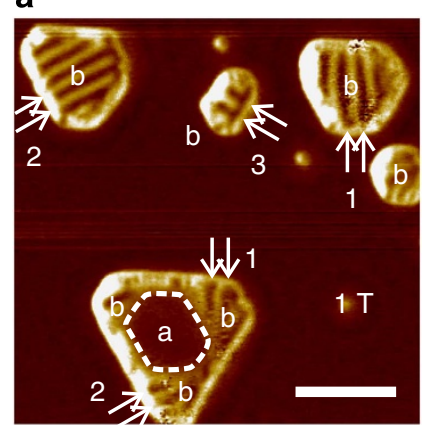

b

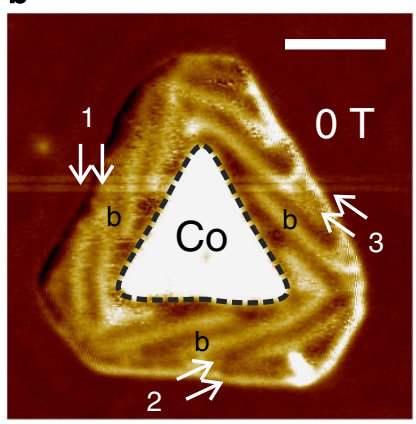

C
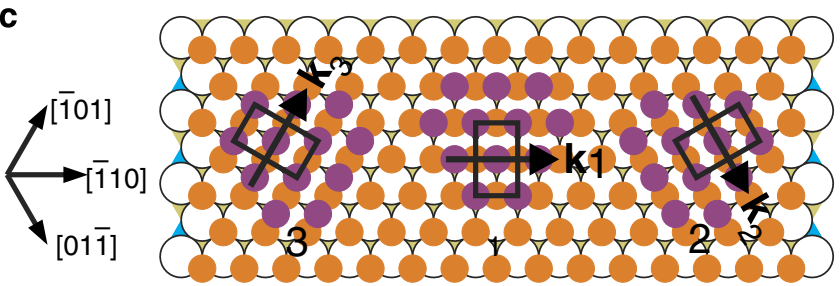

$\bigcirc \mathrm{Cu}$

$\nabla$ Cu hcp site

$\triangle$ Cu fcc site magnetization of the Co core along the out-of-plane direction fixes the $\mathrm{Fe}$ spin orientation at the $\mathrm{Co}-\mathrm{Fe}$ border by exchange coupling $^{9}$, and this ascertains a unique phase of the stripe contrast in each Fe-b region.

Magnetic-field-dependent differential conductance of Fe-b. A spatially periodic magnetic state could originate from either a collinear spin-density wave ${ }^{27,28}$ (SDW) or a helical SDW ${ }^{6}(\mathrm{SH})$. A collinear SDW is characterized by a periodic change of the magnitude of the spin moments, while the spin orientation is fixed. On the other hand, a SH is composed of spin moments of constant magnitude but with changing orientation. To resolve the origin of the spin ordering in the stripe patterns of the Fe-b phase, we perform SP-STS with a tip, where the direction of the tip magnetization $\mathbf{M}_{\mathrm{T}}$ can be tuned by the magnetic field ${ }^{6}$. The magnetic characterization of our tip reveals that its magnetization responds to the external magnetic field like a Stoner-Wohlfarth magnet ${ }^{29}$ with the magnetic easy axis canted by $55 \pm 1^{\circ}$ from the sample normal (Supplementary Fig. 1; Supplementary Note 2). Therefore, the tip used here is sensitive to both in-plane and outof-plane components of the sample magnetization at $0 \mathrm{~T}$, while it is sensitive only to the out-of-plane component of the sample magnetization at large field $(\sim \pm 1.5 \mathrm{~T})$. The $\mathrm{d} I / \mathrm{d} V$ signal in SP-STS depends on $\mathbf{m}_{\mathrm{T}} \cdot \mathbf{m}_{\mathrm{S}}$, where $\mathbf{m}_{\mathrm{T}}$ and $\mathbf{m}_{\mathrm{S}}$ are the unit vectors along the directions of tip and sample magnetizations, respectively ${ }^{25}$. Accordingly, the magnetic-field-dependent differential conductance measurement for a collinear SDW will result only in a change of magnitude but not in the phase of the spatial dependence of the $\mathrm{d} I / \mathrm{d} V$ data. In contrast, the $\mathrm{SH}$ will show a field-dependent phase shift of the stripe pattern of the differential conductance signal, since the tip gets more sensitive to the out-of-plane component of the sample magnetization as the magnetic field increases.

Figure $3 \mathrm{a}$ shows the differential conductance image of an $\mathrm{Fe} \mid \mathrm{Co}$ island, measured at $0 \mathrm{~T}$. The $\mathrm{Fe}-\mathrm{b}$ regions at the three corners of the island show a stripe contrast along three different directions as indicated by the solid lines superposed along the stripes with the labels $1-3$. Figure $3 \mathrm{c}-\mathrm{e}$ shows the field dependences of the differential conductance profiles along the lines $\mathrm{AA}^{\prime}, \mathrm{BB}^{\prime}$ and $\mathrm{CC}^{\prime}$ perpendicular to the three directions of the stripe patterns in Fig. 3a, respectively. The wavelengths of the stripe patterns for all three directions, as denoted in Fig. 3c-e, are identical within error margins of $\pm 0.1 \mathrm{~nm}$, and exhibit the same value of $1.28 \mathrm{~nm}$ as observed in the pure Fe island (Fig. 1c,d). Figure $3 \mathrm{~b}$ shows a zoom-in of the field dependence of the two peaks in the profiles along the line $\mathrm{AA}^{\prime}$, as shown within the grey dashed line in Fig. 3c, for clarity. With increasing magnetic field, the positions of maxima and minima move monotonically from right to left, while the distance between the extrema remains constant. A phase shift of $\Delta P \sim 0.18 \mathrm{~nm}$ is measured upon a change of the magnetic field from $0-1.5 \mathrm{~T}$. This observation rules out a collinear SDW. Rather, the in-field SP-STS identifies a SH as the spin ordering of the stripe contrast in the Fe-b phase. The extrema of stripe patterns at three corners of the island, with increasing field, show different amounts of phase shifts. We perform a quantitative analysis of the phase shift $\Delta P$ and its dependence on stripe orientation and field (Supplementary Fig. 2; Supplementary Note 3). An excellent agreement between the experiments and simulations (Supplementary Fig. 3; Supplementary Note 4) reveals that the field dependences of the phase shifts are determined by a given orientation of the tip magnetization.

Theoretical study of $\mathrm{SH}$ in $\mathrm{BL}$ Fe on $\mathrm{Cu}(111)$. The wavelength of the $\mathrm{SH}(1.28 \mathrm{~nm})$ corresponds to only about five surface lattice 
$a$

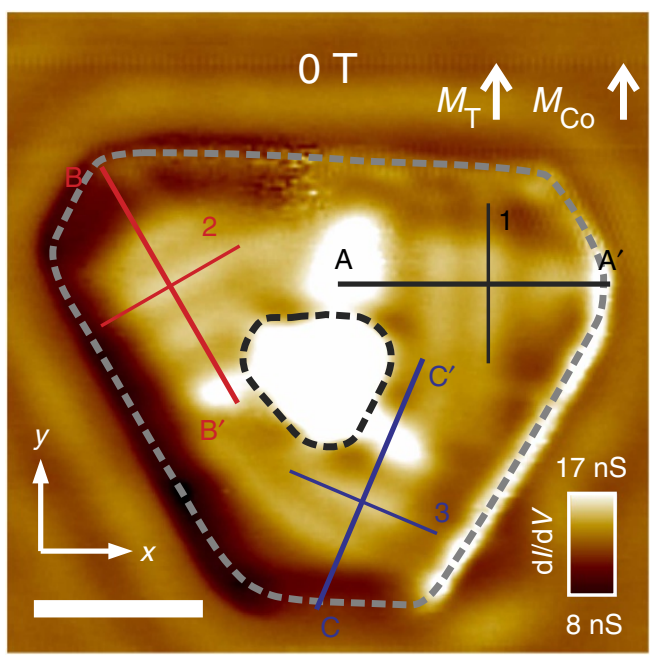

b
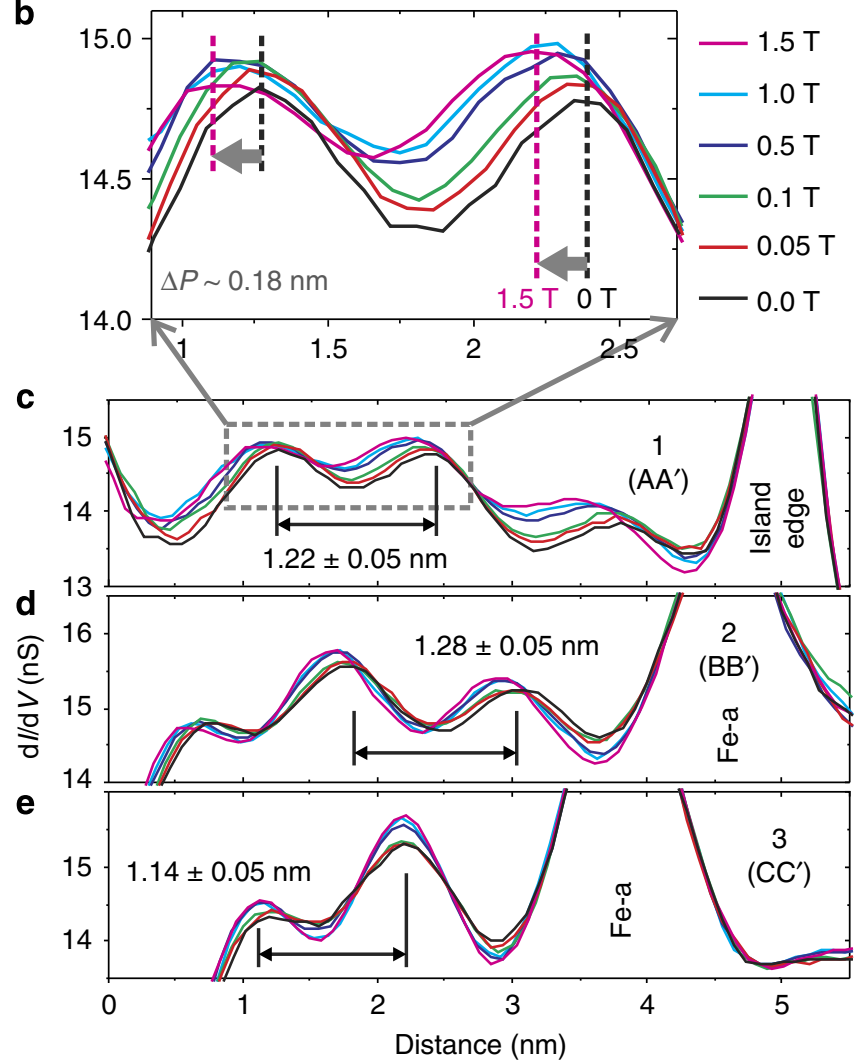

Figure 3 | Field-dependent differential conductance signals in Fe-b phases. (a) Differential conductance $\mathrm{d} / / \mathrm{d} V$ map of an Fe|Co island measured at OT, where both magnetizations of tip $\mathbf{M}_{\mathrm{T}}$ and Co core $\mathbf{M}_{\mathrm{Co}}$ point up $\left(V_{b}=-0.3 V, I_{\text {set }}=3 n A\right)$. The island edges and Co core are indicated by the grey and black dashed lines. The black, red and blue solid lines (arrows) indicate the directions of the stripes (wave vectors of the stripe patterns), corresponding to the Fe-b regions 1, 2 and 3, respectively. (c-e) Field-dependent differential conductance $d / / d V$ profiles along the arrows $\mathbf{1}$ (c), $\mathbf{2}$ (d) and $\mathbf{3}$ (e) in a, measured with the field sweep from 0-1.5 T. (b) Magnified view of the grey dashed box in $\mathbf{c}$ and is shown for a clarity of field-dependent peak position shifts in $\mathbf{c}$. The black and purple dotted lines denote the peak positions of the profiles measured at 0 and $1.5 \mathrm{~T}$, respectively. Scale bar in a indicates $4 \mathrm{~nm}$. constants of $\mathrm{Cu}$ along $\langle 110\rangle$. It results in an angle of $\approx 36^{\circ}$ between NN spin moments. This is striking since such a large canting angle gives rise to a dramatic increase in the $\mathrm{NN}$ exchange energy. Consequently, another interaction is operative, which compensates this cost of exchange energy. To solve this puzzle, we apply noncollinear spin-density functional theory (see Methods section) to determine the magnetic energy for BL Fe on $\mathrm{Cu}(111)$ with the topmost $\mathrm{Fe}$ in a bridge position, as illustrated in the inset of Fig. 4a. Figure 4a shows the SH formation energy $E_{\mathrm{SH}}-E_{\mathrm{FM}}$ as a function of the wave vector $k_{\overline{1} 10}$, at three different $\mathrm{Fe}-\mathrm{Fe}$ interlayer distances, where $d=2.10 \AA$ indicates the FM state of the system (Supplementary Methods). First, we calculated the formation energy of a SH state, where the SOI was not taken into account (circles). Interestingly, at reduced interlayer distances-which are expected for the $\mathrm{BL} F e$ on $\mathrm{Cu}(111)$ - energy minima (of $\Delta E \sim 10 \mathrm{meV}$ per atom) develop. The minima get more pronounced for smaller $d$. The wave vector $k_{\overline{1} 10}=0.9 \pm$ $0.1 \mathrm{~nm}^{-1}$ at the energy minima corresponds to a $\mathrm{SH}$ wavelength of $1.1 \pm 0.15 \mathrm{~nm}$, which is in favourable agreement with the experimental observation. Next, we calculate the $\mathrm{SH}$ formation energies including SOI and superimpose the results (crosses) in Fig. 4a. The SOI does not lead to any notable contribution to the total SH formation energy. We show the contribution of SOI to the total $\mathrm{SH}$ formation energy $E_{\mathrm{SH}}^{(\mathrm{SOI})}-E_{\mathrm{SH}}^{(0)}$ in Fig. $4 \mathrm{~b}$, where $E_{\mathrm{SH}}^{(\mathrm{SOI})}$ and $E_{\mathrm{SH}}^{(0)}$ represent the energies calculated with and without SOI, respectively. The size of SOI energy is less than $\sim 0.5 \mathrm{meV}$ per atom at $k_{\overline{1} 10}$ of the energy minima. This is by a factor of order 20 smaller than the energy minima shown in Fig. 4a. To account for a possible impact of in-plane structural relaxation in the Fe-b phase proposed in an earlier report ${ }^{21}$, we also performed the same calculations for free-standing $\mathrm{BL} F$ systems of a bridge-site interlayer stacking with two different sets of lattice constants (Supplementary Fig. 4; Supplementary Note 5). Surprisingly, the effect of a reduced interlayer distance $d$ is more pronounced. The in-plane relaxation even lowers the $\mathrm{SH}$ energy minima from those of the unrelaxed structure by $\sim 20 \mathrm{meV}$ per atom (Supplementary Fig. 4; Supplementary Note 6).

\section{Discussion}

Our SP-STM/S observations indicate a novel, previously not described, helical spin order stabilized in a single nanostructure, which is magnetically decoupled from the substrate. This is in contrast to previously reported SHs in ultrathin magnetic films on $\mathrm{W}$ and $\mathrm{Ir}^{6,8,9}$, where a sizable $\mathrm{DMI}^{14,15}$, induced by a large SOI at the film-substrate interface, has been identified as the origin of the $\mathrm{SH}$ formation. Our $a b$ initio calculations exclude the role of SOI on the formation energy of our SH. The negligible contribution of SOI in the Fe-b system is ascribed to the small spin-orbit coupling strength of the $3 d$ elements $\mathrm{Fe}$ and $\mathrm{Cu}$, which is expected to be an order of magnitude weaker than that of $5 d$ components $^{30}$ (for example, $\mathrm{W}$ or Ir). The compilation of the $J_{\mathrm{i}, \mathrm{j}}$ elucidates the physical origin of $\mathrm{SH}$ formation in this system. We extract interatomic exchange interaction parameters $J_{\mathrm{i}, \mathrm{j}}$ for a $\mathrm{SH}$ state in this system by applying one-dimensional effective Heisenberg model to the calculated $\mathrm{SH}$ formation energies. Figure $4 \mathrm{c}$ shows the interspin distance-dependent $J_{\mathrm{i}, \mathrm{j}}$, extracted from the $E_{\mathrm{SH}}-E_{\mathrm{FM}}$ values of $d=2.01 \AA$ (red circles) in Fig. $4 \mathrm{a}$, between $i=0$ th and $j=1$ st to 10 th rows along the $[\overline{1} 10]$ direction as indicated in the inset. The $J_{01}$ is a positive value of $\sim 108 \mathrm{meV}$, which means that it favours a FM NN spin alignment. On the other hand, the $J$ s from the second to the fifth neighbour $\left(J_{02}-J_{05}\right)$ are negative, and this favours AFM spin alignments. These sizable AFM Js of the long-distant neighbours ${ }^{13}$ reflect the lowering of the energy by the helical arrangement of the spin moments in Fe-b within the Heisenberg model. 
a

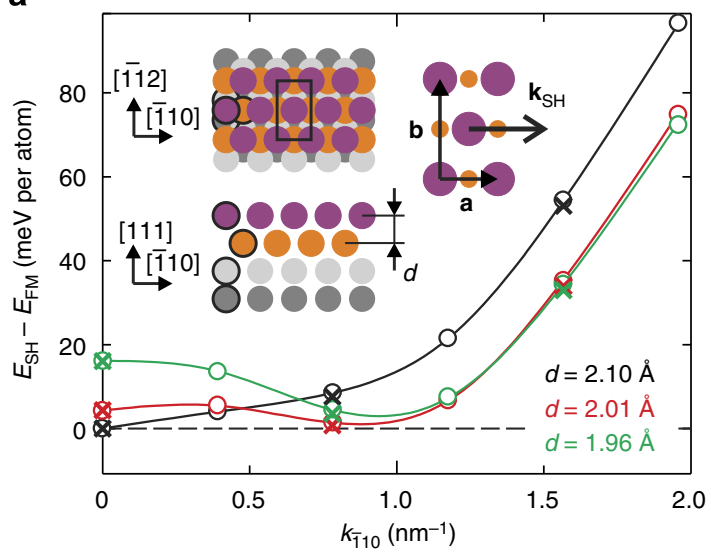

b

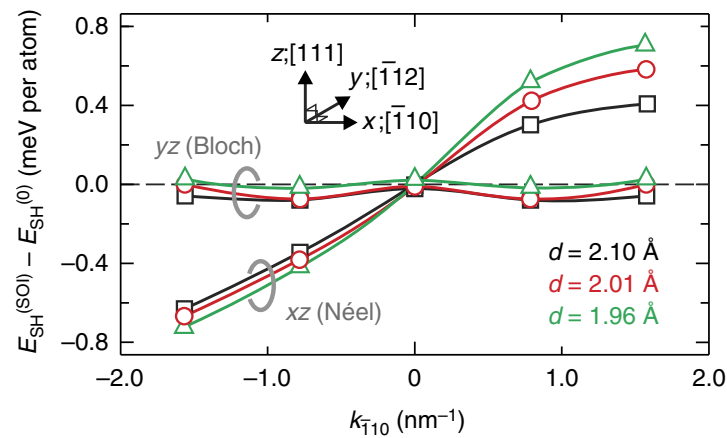

C

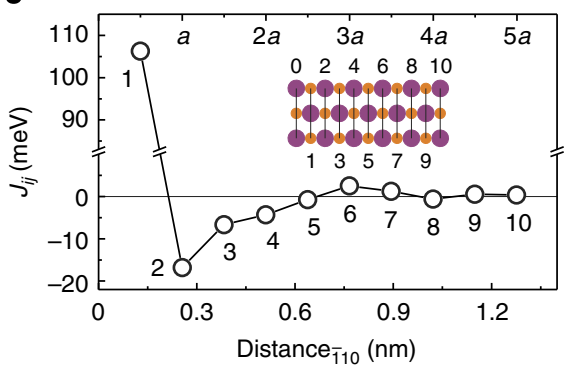

d

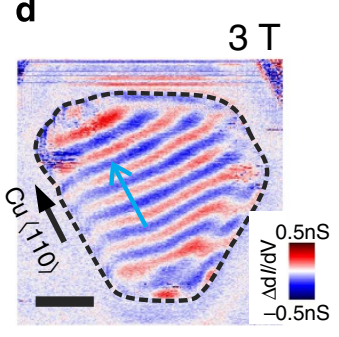

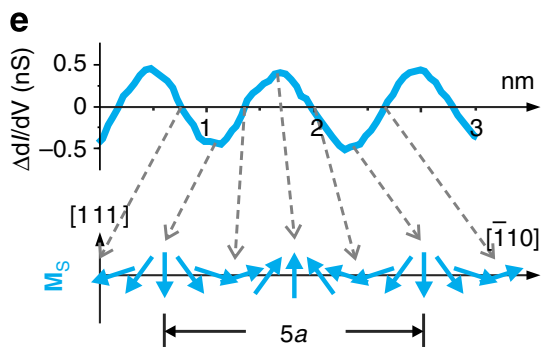

Figure 4 | Calculated formation energy $\left(\boldsymbol{E}_{\mathbf{S H}}-\boldsymbol{E}_{\mathbf{F M}}\right)$ and magnetic ordering of SH in the Fe-b phase. (a) Circles are the calculated formation energies of $\mathrm{SH}$ without $\mathrm{SOI}$, as a function of wave vector $k_{\mathrm{SH}}$ along the $\mathrm{SH}$ direction observed in the experiment at the Fe-Fe interlayer distances $d=2.10,2.01$ and $1.96 \AA$. The structure of the calculation model, a BL Fe on $\mathrm{Cu}(111)$ with topmost Fe in bridge-site-stacking, is depicted by a hard sphere model (top and side views, surface unit cell) in the inset. ( $\boldsymbol{a}$ and $\boldsymbol{b}$ ) are the surface lattice vectors along the $[110]_{\mathrm{fcc}}$ and $[112]_{\mathrm{fcc}}$ directions, respectively. Here the surface lattice constants of BL Fe of an exact epitaxy on $\mathrm{Cu}(111)(a=2.55 \AA$ and $b=4.42 \AA)$ are used. The reference (zero) energy is chosen for the FM state $E_{\mathrm{FM}}$ $\left(k_{\mathrm{SH}}=0\right)$, which results in $d=2.10 \AA$. Crosses represent the calculated formation energies of SH with SOI, and are superimposed on the curves. (b) Energy difference of the $\mathrm{SH}$ structures with $\left(E_{\mathrm{SH}}^{(\mathrm{SOI})}\right)$ and without $\left(E_{S H}^{(0)}\right) \mathrm{SOI}, E_{\mathrm{SH}}^{(\mathrm{SOI})}-E_{S H}^{(0)}$, at $d=2.10,2.01$ and 1.96 $\AA$. The labels $x z$ and $y z$ indicate the planes of the atomic spin vectors in an SH (thus, Néel- and Bloch-type spin moment arrangements, respectively), where the directions $x, y$ and $z$ are taken as $[110]_{\text {fcc }}$ $[112]_{\mathrm{fcc}}$ and [110], respectively. Note that an asymmetry with respect to the $k_{\overline{110}}$ appears in the results for $x z$ case by an introduction of SOI. (c) Exchange interaction parameters $J_{i j}$, for $i=0$ and $j=1-10$ as indicated in the inset, extracted by applying the one-dimensional (along [110] direction) effective Heisenberg model to $E_{\mathrm{SH}}-E_{\mathrm{FM}}$ with $d=2.01 \AA$ for the non-relativistic case (red circles) in a,d. Pure magnetic-field-induced variation of the $\mathrm{d} / / \mathrm{d} V$ signal $\Delta \mathrm{d} l / \mathrm{d} V$ in the Fe island shown in Fig. 1a, extracted by the subtraction of the $\mathrm{dl} / \mathrm{d} V$ map at OT (Fig. 1b) from that at $3 \mathrm{~T}$ (Fig. 1c). The black dashed curve denotes the island edge. The magnetic response $\Delta \mathrm{d} l / \mathrm{d} V$ here reflects only the position dependence of the out-of-plane component of the sample magnetization $\mathbf{M}_{\mathrm{S}}$ since the tip magnetization $\mathbf{M}_{\mathrm{T}}$ is aligned to the direction of the external magnetic field (Supplementary Fig. 1; Supplementary Note 2). Scale bar indicates $3 \mathrm{~nm}$. (e) Position dependence of local magnetization (cyan arrows) is sketched with the $\Delta \mathrm{dl} / \mathrm{dV}$ profile along the arrow in $\mathbf{d}$.

Although its magnitude is minute, a quantitative discussion of SOI in this system provides insights into the local magnetic ordering of the $\mathrm{SH}^{12}$. An interesting point is that a $\mathrm{SH}$ of the spin vectors rotating in the $x z$ plane gives rise to an asymmetry in $E_{\mathrm{SH}}^{(\mathrm{SOI})}-E_{\mathrm{SH}}^{(0)}$ about $k_{\overline{1} 10}=0$. This spin structure contributes a much larger SOI energy with a positive (negative) sign, as compared with a $\mathrm{SH}$ rotating in the $y z$ plane, for a positive (negative) wave vector. The asymmetry in SOI with respect to $k_{\mathrm{SH}}$ is ascribed to the contribution of the spatially antisymmetric $\mathrm{DMI}^{14,15}$ (Supplementary Discussion). The inspection of SOI in this system reveals that a spin rotation in the $x z$ plane with a negative $k_{\mathrm{SH}}$ is energetically favoured, giving rise to a Néel-type spin rotation with left-handed chirality, as depicted in Fig. 4e.

To understand the absence of magnetic stripe contrast at zerofield in a pure Fe island (Fig. 1b), it is instructive to consider the relevant energy scale. The tip shows a non-zero magnetization at $0 \mathrm{~T}$ (Supplementary Fig. 1; Supplementary Note 2). Thus, we ascribe the absence of zero-field spin-contrast to coherent fluctuation of the spin order in the $\mathrm{SH}$, which are fast on the timescale of STM (several ten seconds). Thus, we speculate that the $\mathrm{SH}$ is present at $0 \mathrm{~T}$; however, its phase fluctuates, rendering it invisible to the SP-STM/S. The Zeeman energy $E_{\mathrm{Zee}}$ at $3 \mathrm{~T}$ amounts to only $0.38 \mathrm{meV}$ per atom (Supplementary Discussion). This is smaller by a factor of order 300 (30) than the NN (next $\mathrm{NN}$ ) exchange energy of the $\mathrm{SH}$ in our theoretical prediction. This reveals that our magnetic fields are by far too weak to modify the metric of the SH. However, still, a moderate field is sufficient to stabilize the phase of the SH. This is ascribed to the magnetic field interacting with uncompensated spin moments of $\mathrm{SH}$. The finite lateral size of the island may work as a factor in determining the unique phase of the $\mathrm{SH}$ in the following way: a $\mathrm{SH}$ length $l_{\mathrm{SH}} \neq n \lambda_{\mathrm{SH}}$, where $n=$ integer, induces uncompensated net magnetic moment at either end of a SH. This uncompensated moment tends to align its direction parallel to the external magnetic field, providing stabilization of the phase in magnetic fields. In addition, a higher spin state ${ }^{5,31}$ and larger susceptibility of the spins at the island edge would facilitate this process further.

Moreover, the observation of the stripe contrast at $0 \mathrm{~T}$ in $\mathrm{Fe}-\mathrm{b}$ regions of an $\mathrm{Fe} \mid \mathrm{Co}$ island excludes the role of the $E_{\mathrm{Zee}}$ on the formation of $\mathrm{SH}$. Hence, we neglect the role of $E_{\mathrm{Zee}}$ on the fielddependent changes in the differential conductance shown in Fig. 3c-e. The estimation of the magnetic anisotropy (MA) energy 
$E_{\mathrm{MA}}$ of the Fe-b structure, from a hypothetically calculated FM state $\left(d=2.10 \AA ; k_{\mathrm{SH}}=0\right)$, favours a MA along the out-of-plane direction [111 $]_{\mathrm{fcc}}$. The energy gain is 0.33 and $0.24 \mathrm{meV}$ per atom as compared with magnetization along two primary in-plane directions $[\overline{1} 10]_{\mathrm{fcc}}$ and $[\overline{1} 12]_{\mathrm{fcc}}$, respectively (Supplementary Discussion). The thermal energy $E_{\mathrm{th}}$ at our measurement temperature of $10 \mathrm{~K}$ is $0.86 \mathrm{meV}$ (Supplementary Discussion). All these energy scales are by far too small to form or change the internal spin structure of the observed SH in this study, which is because of orders of magnitude larger $J_{\mathrm{ij}}$. This supports the view that the formation of the $\mathrm{SH}$ in Fe-b is driven by a subtle interplay of the exchange interaction acting differently at different spinspin distances. Thermal energy ${ }^{32}$ or zero point energy ${ }^{33}$ may drive the fluctuation of the phase of the $\mathrm{SH}$, which results in an averaged-out magnetic signal in SP-STM/S at $0 \mathrm{~T}$.

In conclusion, our combined experimental and theoretical studies on the BL Fe nanoisland on $\mathrm{Cu}$ (111) reveal a helical spin order in a system, where SOI and magnetic interactions with the substrate are negligible. This spin order is driven by sizable longdistance AFM exchange interactions, which arise from nanosizedriven structural relaxations. The observation of helimagnetism in Fe nanostructures on a light $3 d$-element substrate sheds fresh light on the importance of competing exchange interactions on different length scales, arising from the reduced dimensionality of the nanostructures, as a driving force of noncollinear spin states. Our study opens the way for alternative venues to produce noncollinear spin states for potential applications of the MNs.

\section{Methods}

Experiments. The experiments were performed in an ultrahigh vacuum (UHV) chamber (base pressure $<1 \times 10^{-11} \mathrm{mbar}$ ) equipped with a STM operating at $10 \mathrm{~K}$ and a superconducting magnet for magnetic field of up to $7 \mathrm{~T}$, normal to the sample surface. The $\mathrm{Cu}(111)$ single crystal substrate (MaTeck $\mathrm{GmbH})$ was cleaned by the repetition of $\mathrm{Ar}^{+}$-sputtering $(1 \mathrm{keV}, 0.75 \mu \mathrm{A}$ sample current and $15 \mathrm{~min}$ per cycle) and subsequent heating at $700 \mathrm{~K}$ for $15 \mathrm{~min}$ until defect-free, atomically flat and clean, and large $(>200 \mathrm{~nm}$ ) terraces are observed in STM. We deposit 0.24-ML Co, and then, subsequently, 0.28-ML Fe on the cleaned $\mathrm{Cu}$ surface at $300 \mathrm{~K}$ in UHV. The sequential deposition leads to the formation of two different types of islands on the $\mathrm{Cu}$ substrate; $\mathrm{Fe} \mid \mathrm{Co}$ island and isolated $\mathrm{Fe}$ island, both are BL high. STM and STS on an Fe|Co island revealed that the distinction between Co and two different Fe structural phases is possible by a difference in apparent heights and pronounced spectroscopic signatures in the differential conductance ${ }^{26}$. In order to obtain magnetic contrast, we used $\mathrm{Cr} / \mathrm{Co}$-coated $\mathrm{W}$ tips. To obtain a $\mathrm{Cr} / \mathrm{Co}$-coated W tip, we deposited 40 atomic layers of Co and 40 atomic layers of $\mathrm{Cr}$ subsequently on an electrochemically etched $\mathrm{W}$ tip, which was briefly heated in $\mathrm{UHV}$ up to $2,400 \mathrm{~K}$ for $2 \mathrm{~s}$ before the depositions. Then, the $\mathrm{Cr} / \mathrm{Co}$-coated $\mathrm{W}$ tip was annealed at $600 \mathrm{~K}$ for $5 \mathrm{~s}$. We employed a lock-in technique with a modulation bias voltage at a frequency $v=4 \mathrm{kHz}$ and of a root-mean square amplitude of $20 \mathrm{mV}$ to detect the tunnel current $I(V)$ and the differential conductance $\mathrm{d} I / \mathrm{d} V$ simultaneously. Point spectroscopy is performed by positioning the tip at the position of STS measurement and before switching the feedback loop off, we stabilize the tip-sample junction at the bias voltage $V_{\text {Stab }}=0.5 \mathrm{~V}$ and the tunnelling current $I_{\text {Stab }}=1 \mathrm{nA}$.

Theory. Density functional calculations were performed based on generalized gradient approximation by using full-potential linearized augmented plane-wave method in which the valence states are treated scalar-relativistically, that is, without SOI, and generalized Bloch theorem is applied for helical spin structures. The SOI was included by the second variational method. Calculations were carried out by: first allowing the interlayer distances in the film to relax by atomic force calculations within the FM state, then the formation energy of the SH state is obtained by varying the $\mathrm{Fe}-\mathrm{Fe}$ interlayer distance. We extract the exchange interaction parameter $J_{\mathrm{ij}}$ for an interatomic row (along $[\overline{1} 12]$ direction) by applying the back Fourier transformation of the calculated $\mathrm{SH}$ formation energy $E_{\mathrm{SH}}-E_{\mathrm{FM}}$ with one-dimensional effective Heisenberg model along the $[\overline{1} 10]_{\text {fcc }}$ direction. More details of the calculation are given in the Supplementary Methods.

\section{References}

1. Mühlbauer, S. et al. Skyrmion lattice in a chiral magnet. Science 323, 915-919 (2009).

2. Yu, X. et al. Real-space observation of a two-dimensional skyrmion crystal. Nature 465, 901-904 (2010).
3. Kurz, P. h., Bihlmayer, G., Hirai, K. \& Blügel, S. Three-dimensional spin structure on a two-dimensional lattice: $\mathrm{Mn} / \mathrm{I} z ̌ \mathrm{Cu}(111)$. Phys. Rev. Lett. 86, 1106-1109 (2001).

4. Bergman, A. et al. Magnetic interactions of Mn clusters supported on $\mathrm{Cu}$. Phys. Rev. B 73, 174434 (2006).

5. Bergman, A., Nordström, L., Klautau, A. B., Frota-Pessôa, S. \& Eriksson, O. Magnetic structures of small $\mathrm{Fe}, \mathrm{Mn}$, and $\mathrm{Cr}$ clusters supported on $\mathrm{Cu}(111)$ : noncollinear first-principles calculations. Phys. Rev. B 75, 224425 (2007).

6. Bode, M. et al. Chiral magnetic order at surfaces driven by inversion asymmetry. Nature 447, 190-193 (2007).

7. Heinze, S. et al. Spontaneous atomic-scale magnetic skyrmion lattice in two dimensions. Nat. Phys. 7, 713-718 (2011).

8. Ferriani, P. et al. Atomic-scale spin spiral with a unique rotational sense: Mn monolayer on W(001). Phys. Rev. Lett. 101, 027201 (2008).

9. Menzel, M. et al. Information transfer by vector spin chirality in finite magnetic chains. Phys. Rev. Lett. 108, 197204 (2012).

10. Braun, H.-B. et al. Emergence of solition chirality in a quantum antiferromagnet. Nat. Phys. 1, 159-163 (2005).

11. Lizárraga, R. et al. Conditions for noncollinear instabilities of ferromagnetic materials. Phys. Rev. Lett. 93, 107205 (2004).

12. Nakamura, K., Mizuno, N., Akiyama, T., Ito, T. \& Freeman, A. Spin-spiral structures in free-standing $\mathrm{Fe}(110)$ monolayers. J. Appl. Phys. 99, 08N501 (2006).

13. Mizuno, N., Nakamura, K., Akiyama, T. \& Ito, T. A. Noncollinear magnetism and exchange interaction in spin-spiral structures of thin film $\mathrm{Fe}(110)$. J. Phys. Condens. Matter. 19, 365222 (2007).

14. Dzyaloshinskii, I. E. Thermodynamic theory of 'weak' ferromagnetism in antiferromagnetic substances. Sov. Phys. JETP 5, 1259-1262 (1957).

15. Moriya, T. Anisotropic superexchange interaction and weak ferromagnetism. Phys. Rev. 120, 91-98 (1960).

16. Jensen, J. \& Mackintosh, A. R. Rare Earth Magnetism (Oxford University Press, 1997).

17. Tsunoda, Y. Spin-density wave in cubic $\gamma$-Fe and $\gamma-\mathrm{Fe}_{100-} \mathrm{Co}_{x}$ precipitates in Cu. J. Phys. Condens. Matter. 1, 10427 (1989).

18. Sjöstedt, E. \& Nordström, L. Noncollinear full-potential studies of $\gamma$-Fe. Phys. Rev. B 66, 014447 (2002).

19. Uzdin, S., Uzdin, V. \& Demangeat, C. Magnetic trimer on non-magnetic substrate: from frustration towards non-collinearity. Europhys. Lett. 47, 556 (1999).

20. Gerhard, L. et al. Magnetoelectric coupling at metal surfaces. Nat. Nanotech. 5, 792-797 (2010).

21. Biedermann, A., Rupp, W., Schmid, M. \& Varga, P. Coexistence of fcc- and bcc-like crystal structures in ultrathin Fe films grown on $\mathrm{Cu}(111)$. Phys. Rev. $B$ 73, 165418 (2006).

22. Thomassen, J., May, F., Feldmann, B., Wuttig, M. \& Ibach, H. Magnetic live surface layers in $\mathrm{Fe} / \mathrm{Cu}(100)$. Phys. Rev. Lett. 69, 3831 (1992).

23. Li, D., Freitag, M., Pearson, J., Qiu, Z. Q. \& Bader, S. D. Magnetic phases of ultrathin $\mathrm{Fe}$ grown on $\mathrm{Cu}(100)$ as epitaxial wedges. Phys. Rev. Lett. 72, 3112 (1994).

24. Qian, D., Jin, X. F., Barthel, J., Klaua, M. \& Kirschner, J. Spin-density wave in ultrathin Fe films on $\mathrm{Cu}(100)$. Phys. Rev. Lett. 87, 227204 (2001).

25. Wortmann, D., Heinze, S., Kurz, P., Bihlmayer, G. \& Blügel, S. Resolving complex atomic-scale spin structures by spin-polarized scanning tunneling microscopy. Phys. Rev. Lett. 86, 4132 (2001).

26. Phark, S.-H., Fischer, J. A., Corbetta, M., Sander, D. \& Kirschner, J. Superparamagnetic response of Fe-coated $\mathrm{W}$ tips in spin-polarized scanning tunneling microscopy. Appl. Phys. Lett. 103, 032407 (2013).

27. Overhauser, A. W. Spin density waves in an electron gas. Phys. Rev. 128, 1437-1452 (1962).

28. Fawcett, E. Spin-density-wave antiferromagnetism in chromium. Rev. Mod. Phys. 60, 209-283 (1988).

29. Stoner, E. \& Wohlfarth, E. A mechanism of magnetic hysteresis in heterogeneous alloys. Phil. Trans. R. Soc. A 240, 599-642 (1948).

30. Chen, G. et al. Novel chiral magnetic domain wall structure in $\mathrm{Fe} / \mathrm{Ni} / \mathrm{Cu}(001)$ films. Phys. Rev. Lett. 110, 177204 (2013).

31. Újfalussy, B., Szunyogh, L. \& Weinberger, P. Magnetic anisotropy in $\mathrm{Fe} / \mathrm{Cu}(001)$ overlayers and interlayers: the high-moment ferromagnetic phase. Phys. Rev. B 54, 9883 (1996).

32. Krause, S. et al. Magnetization reversal of nanoscale islands: how size and shape affect the Arrhenius prefactor. Phys. Rev. Lett. 103, 127202 (2009).

33. Holzberger, S., Schuh, T., Blügel, S., Lounis, S. \& Wulfhekel, W. Parity effect in the ground state localization of antiferromagnetic chains coupled to a ferromagnet. Phys. Rev. Lett. 110, 157206 (2013).

\section{Acknowledgements}

This work was partially supported by Deutsche Forschungsgemeinschaft grant SFB 762 . We gratefully acknowledge the discussion with S. Ouazi.

\section{Author contributions}

S.-H.P., D.S. and J.K. conceived and designed the experiments. S.-H.P., J.A.F. and M.C. prepared samples and performed spin-polarized STM and STS measurements. S.-H.P. 
analysed spin-polarized STM and STS data. K.N. performed the $a b$ initio calculation. All authors discussed the results and wrote the paper.

\section{Additional information}

Supplementary Information accompanies this paper at http://www.nature.com/ naturecommunications
Competing financial interests: The authors declare no competing financial interests.

Reprints and permission information is available online at http://npg.nature.com/ reprintsandpermissions/

How to cite this article: Phark, S.-H. et al. Reduced-dimensionality-induced helimagnetism in iron nanoislands. Nat. Commun. 5:5183 doi: 10.1038/ncomms6183 (2014). 